\title{
ÉtICA PROFESIONAL Y PROFESORES DE POSGRADO EN MÉXICO ${ }^{\star}$
}

Ana Hirsch Adler ${ }^{\star \star}$

\begin{abstract}
RESUMEN
Se presentan los resultados obtenidos con una muestra de 704 profesores de los cuarenta posgrados de la Universidad Nacional Autónoma de México (UNAM), con la pregunta abierta ¿Considera usted que debería existir una materia de ética profesional para todo el alumnado? - ¿Con qué contenidos?. Las múltiples respuestasseorganizaron con baseen dosclasificaciones: tres grupos: "Respuestas no directamente ligadas con la ética profesional", "Valores vinculantes entre la ética general y la ética profesional" y "Respuestas directamente ligadas con la ética profesional" y las cuatro grandes áreas de conocimiento en que la UNAM clasifica sus posgrados: Ciencias Físico Matemáticas y de las Ingenierías, Ciencias Biológicas y de la Salud, Ciencias Sociales y Humanidades y de las Artes. La pregunta forma parte de un proyecto de investigación sobre ética profesional y de un cuestionario que incluye otras preguntas abiertas y cerradas y una escala de actitudes sobre ética profesional.
\end{abstract}

Palabras clave: profesores de posgrado; ética profesional; áreas de conocimiento; México; Universidad Nacional Autónoma de México.

\section{Professional Ethics and Posgraduate Professors in Mexico}

\begin{abstract}
We present the results generated with a sample of 704 professors of the forty graduate programs in the National Autonomous University of Mexico (UNAM), with the open question: Do you consider that it should exist a subject about professional ethics for all students?, With which contents?. The multiple answers were organized with two types of classification: three groups - "Answers not directly related to professional ethics", "Articulating values among general ethics and professional ethics" and "Answers directly related to professional ethics"

\footnotetext{
^ Organismo de financiación: Programa de Apoyo a Proyectos de Investigación e Innovación Tecnológica de la UNAM

$\star \star$ Investigadora Titular "C" en el Instituto de Investigaciones sobre la Universidad y la Educación de la Universidad Nacional Autónoma de México. Dirección: Universidad Nacional Autónoma de México, Edificio IISUE, Ciudad Universitaria, Centro Cultural Universitario, México Distrito Federal, Coyoacan 04510.

Correo electrónico: anaha007@yahoo.com.mx
} 
- and the four knowledge areas in which the university organize the graduate studies: Physical and Mathematics Sciences and Engineering, Biological and Health Sciences, Social Sciences and Humanities and the Arts. The open question is part of a research project about professional ethics and of a questionnaire that includes other questions and an attitude scale about professional ethics.

Keywords: posgraduate professors; professional ethics; knowledge areas; Mexico; National Autonomous University of Mexico.

\section{INTRODUCCIÓN}

Este trabajo se sustenta en varias afirmaciones:

- Hay un predominio de contenidos cognitivos y técnicos en la formación profesional de los estudiantes universitarios; lo que deja generalmente de lado la inclusión de contenidos, competencias y habilidades de carácter ético, social y afectivo - emocional.

- La ética profesional es un aspecto significativo y necesario en la formación integral de los estudiantes; que puede enseñarse de manera explícita. ${ }^{1}$

- La ética profesional es una ética aplicada, que se distingue de la ética en general y de la ética cívica, pues posee su propio marco teórico de referencia, valores específicos y experiencias particulares. ${ }^{2}$

Se retoma para este artículo, la contestación a la pregunta: ¿Considera usted que debería existir una materia de ética profesional para el alumnado en todas las instituciones?. En caso afirmativo ¿Con qué contenidos?.

Para contestarla nos basamos en la codificación de todas las respuestas a esta pregunta abierta, por parte de una muestra de 704 profesores de los cuarenta posgrados de la Universidad Nacional Autónoma de México (UNAM) en 2006 y 2007.

\section{EL PROYECTO DE INVESTIGACIÓN SOBRE ÉTICA PROFESIONAL DE LA UNAM}

Los dos principales objetivos del proyecto de investigación sobre ética profesional son: contribuir a la generación de conocimiento sobre la ética profesional universitaria en el nivel del posgrado y proponer lineamientos para la formación en ética profesional. Desde su construcción interesaba diferenciar los principales rasgos de ética profesional de los estudiantes y profesores de posgrado de acuerdo a sus áreas de conocimiento.

La investigación se lleva a cabo en el Instituto de Investigaciones sobre la Universidad y la Educación de la UNAM y cuenta con tres fases.

En la primera se realizaron cinco tipos de actividades interrelacionadas: construcción del marco teórico, ${ }^{3}$ construcción y actualización de un estado de conocimiento sobre las investigaciones sobre valores profesionales en México, once entrevistas con profesores de algunas universidades españolas, construcción 
de una escala de actitudes sobre ética profesional y su aplicación en dos muestras de grupos de alumnos de posgrado, una en España en quince posgrados, en las Universidades de Valencia, Politécnica de Valencia y Murcia y otra, en México, en los 40 posgrados de la UNAM.

En la segunda fase, se aplicó el mismo instrumento a una muestra de profesores de los 40 posgrados de la UNAM en 2006 y 2007. En la última fase, en 2009, se aplicó una guía de entrevista abierta a los cuarenta coordinadores de posgrado de la UNAM. Lo obtenido en esta parte final del trabajo empírico, permitirá ampliar la información generada por la pregunta abierta que aquí se describe y analiza.

\section{a) El cuestionario - escal a}

El instrumento que se aplicó en los cuarenta posgrados de la UNAM, a una muestra de 1,086 estudiantes y 719 profesores, ${ }^{5}$ tiene una parte común: la pregunta abierta sobre cuáles son los cinco principales rasgos de ser un buen profesional y una escala de actitudes sobre ética profesional, con 55 proposiciones, especialmente construida para este proyecto.

En el caso de los profesores se agregaron tres preguntas cerradas: Marque el grado de importancia que, a su juicio, hoy se le concede por parte de los académicos universitarios a la ética profesional docente, ¿Qué grado de importancia le concede usted a la ética profesional en su trabajo académico? e Indique ¿en qué grado cree que la universidad, como institución, debe desarrollar los valores profesionales de sus alumnos (as)?.

Se incorporaron también tres nuevas preguntas abiertas que son: Exponga los cinco valores básicos que la universidad debería promover en su alumnado y en su profesorado y ¿Considera usted que debería existir una materia de ética profesional para el alumnado en todas las instituciones? y En caso afirmativo, ¿qué contenidos debería tener esa materia?. El presente trabajo recupera las respuestas a esta última pregunta abierta y las organiza con base en las cuatro áreas de conocimiento en que la UNAM clasifica sus posgrados.

\section{b) La muest ra de profesores de posgrado de I a UNAM}

El $53.40 \%$ son hombres y el $46.59 \%$ mujeres. No es una población joven, pues, en los rangos de edad de 46 a más de 60 años se ubica el $70.38 \%$. Los datos sobre la edad coinciden con la antigüedad académica, pues en el amplio rango de 21 y más de 30 años de servicio se localiza un poco más de la mitad de la muestra (con 50.91\%). Casi la tercera parte de la muestra cuenta con el grado de doctorado $(70.51 \%)$ y con maestría el $24.34 \%$.

En la muestra, hay un $13.35 \%$ de profesores que son contratados para dar clases en asignaturas específicas. En esta universidad se clasifica al personal académico en tres categorías: investigadores, que son los que están adscritos a los institutos y centros de investigación; profesores, que trabajan en las escuelas y facultades y técnicos académicos. A pesar de esta división, muchos de los profesores de las escuelas y facultades también realizan investigación. Los in- 
vestigadores y los profesores con el más alto nivel representaron en la muestra el $13.07 \%$ y el $11.40 \%$ respectivamente. Contestaron el instrumento, también siete eméritos de la UNAM (0.98\%). El resto se coloca de manera dispersa en las otras categorías laborales.

\section{c) Problemas para la clasificación de los profesores por posgrado y por áreas de conocimiento}

Durante el proceso de investigación, nos encontramos con un problema para la ubicación de los académicos por posgrado y por área de conocimiento, porque muchos de ellos trabajan simultáneamente en varios posgrados (de su propia área de conocimiento y de otras). Por ello, se tomó la siguiente resolución: los profesores e investigadores de la muestra se ubicaron en cada uno de los 40 programas y en las cuatro áreas de conocimiento correspondientes ${ }^{6}$ y se generaron otros dos grupos: académicos que sólo dan clases o son tutores en la Maestría en Docencia de la Educación Media Superior, que se ofrece en todas las áreas de conocimiento y los que imparten clases y tutorías en posgrados de dos o más áreas de conocimiento.

En Ciencias Físico Matemáticas y de las Ingenierías ${ }^{7}$ se encuestaron 109 profesores, en Ciencias Biológicas y de la Salud ${ }^{8} 179$, en Ciencias Sociales ${ }^{9} 184$ y en Humanidades y de las Artes ${ }^{10} 143$. En la Maestría en Docencia de la Educación Media Superior fueron 29 y los profesores que están en varios posgrados de diferentes áreas son 74. En este artículo se priorizan las respuestas de los profesores de posgrado en las cuatro áreas de conocimiento.

\section{ReSPUESTAS A LA PREgUNTA DE SI CONSIDERAN QUE DEBERÍA EXISTIR UNA MATERIA DE ÉTICA PROFESIONAL Y CON QUÉ CONTENIDOS}

\section{a) Número de académicos que consideraron necesaria una materia de ética profesional}

La mayoría (75.57\%) indicó que si debería existir una materia de ética profesional para el alumnado.

Los mayores porcentajes de respuestas positivas, por áreas de conocimiento y en orden descendente están en: Ciencias Biológicas y de la Salud (81.87\%), Ciencias Sociales (79.56\%), Maestría en Docencia de la Educación Media Superior (MADEMS) (79.31\%), Humanidades y de las Artes (76.60\%), en dos o más posgrados de diferentes áreas $(71.62 \%)$ y al final en Ciencias Físico Matemáticas y de las Ingenierías (59.26\%).

No sorprende el hecho de que el mayor puntaje de respuestas positivas provenga de las Ciencias Biológicas y de la Salud, que cuentan con varias asignaturas vinculadas con campos interdisciplinarios y de frontera tales como Bioética y Genética y que además han sido siempre pioneros en la reflexión y constitución de leyes internacionales, códigos profesionales, principios y reglas y comités de ética; todo ello de enorme relevancia en el campo de la ética profesional. 
Podemos suponer, que en el área de Ciencias Físico Matemáticas y de las Ingenierías ocupa un lugar preponderante la formación permanente en la ética de las ciencias y de la investigación científica, por lo que los profesores e investigadores de la muestra consideraron poco importante la necesidad de incorporar materias específicas sobre ética profesional.

\section{b) Clasificación de los contenidos sugeridos por los profesores}

El total de las respuestas a los contenidos que debería tener una materia de ética profesional, por parte de los 704 profesores que respondieron la pregunta, fue de 1742. Se trata, pues, de una pregunta detonadora, que generó numerosas y diversificadas respuestas.

Con fines de análisis se clasificaron en tres grupos y 12 categorías.

El primer grupo refiere las "Respuestas no directamente ligadas con la ética profesional", que representa el 39.32\% del total y que incluye cuatro categorías:

- Ética, moral y valores $(25.60 \%$ del total de respuestas).

- Valores cívicos y derechos humanos (6.65\%).

- Historia, cultura y educación (4.93\%), y

- Conducta anticorrupción (2.12\%).

El segundo es el de los "Valores vinculantes entre la ética general y la ética profesional" (que no se subdivide en categorías) y se refiere al 19.51\% del total de las respuestas. Consideramos a estos valores como vinculantes pues en las investigaciones que hemos realizado aparecen mencionados con puntajes altos tanto en aspectos de ética cívica, ética de la ciencia y ética profesional (ALCÁNTARA; BARBA; HIRSCH, 2009, p. 244-246).

El tercero agrupa las "Respuestas directamente ligadas con la ética profesional", aglutina el $41.15 \%$ del total de respuestas y se subdivide en las siguientes ocho categorías:

- Ética Profesional (19.63\% del total).

- Competencias sociales (5.97\%).

- Legalidad y normatividad (4.25\%).

- Identidad (4.19\%).

- Rasgos de las competencias cognitivas (3.55\%).

- Orientación para la toma de decisiones (1.61\%).

- Ética de la investigación científica y tecnológica (1.55\%), y

- Ética docente $(0.40 \%)$. 
Como puede observarse, es escasa la diferencia que hay entre las respuestas no directamente ligadas con la ética profesional y aquellas que si están ligadas directamente con ella. El puente entre ambos grupos: valores vinculantes entre la ética en general y la ética profesional tiene, asimismo, un porcentaje importante.

También es fácil notar que la categoría de Ética, moral y valores, es la que alcanza - con respecto a las 12 categorías de respuesta - el porcentaje más elevado. Esto permite mostrar que aunque el tema central de la investigación es el de ética profesional, los profesores de la UNAM contestaron en alto grado cuestiones generales sobre la ética.

El segundo porcentaje en orden descendente se ubica en la categoría de Ética profesional y en tercer lugar se localizan los Valores vinculantes.

Las otras categorías alcanzan porcentajes mucho más bajos. El que tengan puntajes bajos, no implica, sin embargo, que no sean importantes, como es el caso, por ejemplo de las respuestas ligadas a las conductas anticorrupción.

\section{c) Clasificación de las respuestas por áreas de conocimiento}

A diferencia de lo ya presentado en términos generales, en el área de Ciencias Físico Matemáticas y de las Ingenierías (con un total de 201 respuestas), el grupo más alto es el de las "Respuestas no directamente ligadas a la ética profesional" (44.28\% de las respuestas en esta área de conocimiento). La categoría más numerosa es la de Ética, moral y valores, de la misma manera que en los porcentajes generales, y luego están los "Valores vinculantes entre la ética general y la ética profesional".

Llama la atención que los profesores del Área de Ciencias Biológicas y de la Salud hayan expresado el más alto número de respuestas (459). Presenta resultados diferentes con respecto a la de Ciencias Físico Matemáticas y de las Ingenierías, pues el porcentaje más elevado se ubica en el grupo de "Respuestas directamente ligadas a la ética profesional" (48.80\%) y específicamente en la categoría de Ética profesional (con el 30.28\%). Previamente ya se había mencionado, que en esta área es en donde se ofrecen más materias de ética profesional y que se trata de un campo pionero en la conformación de los principios centrales de la ética profesional.

En Ciencias Sociales, con 399 contestaciones, las más numerosas están en el grupo de "Respuestas no directamente ligadas con la ética profesional" $(45.11 \%)$. La diferencia es mínima, sin embargo, con respecto a las "Respuestas directamente ligadas con la ética profesional" (37.59\%). En cuanto a las categorías, la más alta es Ética, moral y valores.

El Área de Humanidades y de las Artes tuvo 423 respuestas. El mayor porcentaje se ubicó, sorprendentemente en las "Respuestas directamente ligadas con la ética profesional" (43.02\%), aunque de nuevo la categoría mayor fue la Ética, moral y valores. 
En la Maestría en Docencia de la Educación Media Superior y en el caso de los profesores que imparten clases y tutorías en dos o más posgrados de diferentes áreas de conocimiento, se repite lo mismo que en Ciencias Físico Matemáticas y de las Ingenierías y en Ciencias Sociales, en que las respuestas más numerosas se encuentran en el grupo de "Respuestas no ligadas directamente con la ética profesional" y en la categoría de Ética, moral y valores.

De lo encontrado podemos resaltar que la categoría de Ética profesional tiene un porcentaje elevado en las Ciencias Biológicas y de la Salud (se coloca en un primer sitio) y se encuentra en un segundo lugar en todas las demás áreas de conocimiento, exceptuando en Ciencias Físico Matemáticas y de las Ingenierías. La categoría de Ética, moral y valores ocupa el primer lugar en todas las áreas, exceptuando, como ya se mencionó, en Ciencias Biológicas y de la Salud.

\section{Tipos de RESPUESTAS Clasificadas POR ÁREAS DE CONOCIMIENTO}

\section{Área 1. Ciencias Físico Matemáticas y de las Ingenierías}

\section{De corte general y no directamente ligadas con la ética profesional}

a. Ética, Moral y Valores.

- Sobre la ética en general, los contenidos se refieren a: definición, percepción, conceptos y fundamento de la ética y a su relevancia social, ética personal y social, conductas éticas y hábitos positivos, decisiones éticas, relación de la ética con la religión y ética en política.

- Se expresaron temas sobre Filosofía y Axiología, como son: estudio de la Axiología y de los grandes pensadores, énfasis en el análisis de los diferentes sistemas filosóficos y específicamente estudio de la ética estoica y kantiana, el concepto del bien y de lo bueno desde Sócrates hasta Savater y de la ética judeo cristiana, regla de oro, utilitarismo, hedonismo y eudemonismo.

- Sobre la moral se mencionaron: conciencia y acción moral, la moral y las obligaciones del ser humano y la relación entre la ética y la moral.

- Acerca de los valores se indicaron los siguientes asuntos: valores humanos, discusión sobre qué son y para qué sirven los valores, escala de valores y valores individuales, familiares e institucionales.

Resulta enormemente interesante constatar que los profesores de la muestra, en ésta área científica, consideran importante la ética general y que varios de ellos se refieren específicamente a autores y corrientes filosóficas.

b. Valores Cívicos y Derechos Humanos.

Incluye: justicia, igualdad, solidaridad, tolerancia, lealtad, principios de civismo, derechos y responsabilidades de los ciudadanos, considerar el derecho de otros y convivencia de respeto entre individuos, sociedades civiles y autoridades.

c. Historia, Cultura y Educación. 
Los profesores indicaron lo siguiente: principios históricos de la ética, historia de las ideas sobre la ética, su evolución a lo largo del tiempo y su aplicación en el ámbito profesional, historia de la ciencia, la ética y la falta de ésta en la historia de la humanidad e historia de los valores.

También consideraron el estudio del entorno socioeconómico, historia de México, historia reciente de las diferentes clases sociales y económicas de México, temas históricos vinculados a la sociedad y al desarrollo contemporáneo en el planeta, macro y micro economía mexicana, latinoamericana y mundial y aspectos de economía política (intereses del mercado) y su relación con las instituciones de educación e investigación.

e. Conducta anticorrupción.

Aunque se trata de respuestas poco numerosas, son de gran importancia. Se trata de: "contra la corrupción", el soborno y el abuso del poder y la confianza, nepotismo y egoísmo y sus consecuencias.

\section{Valores vinculantes entre la ética en general y la ética profesional.}

Se trata de: responsabilidad, honestidad y honradez, respeto y rectitud e integridad. También hay respuestas ligadas con el compromiso social y ecológico.

\section{Categorías vinculadas directamente con la ética profesional}

\section{a. Ética profesional.}

Se agruparon las siguientes respuestas: qué es la ética profesional en general, ética específica de cada profesión y de profesiones afines, prácticas profesionales basadas en la ética, valores profesionales, el valor del buen profesional, ética laboral, ética en los negocios, la productividad y la ética, ética en el manejo de documentos, cómo conducirse durante el trabajo, cultura laboral que fomente la ética y análisis de la situación actual en diferentes ámbitos profesionales.

b. Competencias y habilidades sociales.

Se mencionan: buen trato con los demás, cómo debe ser la relación con los compañeros y alumnos, capacidad para trabajar en equipo, manejo de conflictos de interés, diálogo, puntualidad, disciplina, orden, dedicación, colaboración, relaciones auténticas y falsas y relaciones humanas.

\section{c. Legalidad, normatividad y principios y reglas de la ética profesional.}

Engloba aspectos como: cumplimiento de normas, principios básicos de la ética profesional, estudio de las leyes que tienen relación con la aplicación de la carrera y las áreas afines, legislación y códigos de la ética profesional, responsabilidad legal, deberes y obligaciones de la profesión, discusión de las normas de ética profesional que las asociaciones imponen o sugieren y respeto y cumplimiento de las reglas profesionales.

d. Identidad. Se dividió en tres grupos de respuestas: 
- Identidad en general, que se refiere a: autovaloración, asertividad, autorrealización, entusiasmo y conciencia de nuestros aspectos positivos y negativos.

- Identidad institucional: discusión y reflexión abierta y propositiva para la situación actual de los estudiantes.

- Identidad profesional: Conciencia de las consecuencias de su profesión, buen cumplimiento de su trabajo, el trabajo como satisfacción espiritual, vocación, vida profesional y satisfacción de servir.

e. Rasgos de las competencias cognitivas.

Incluye: comunicar las habilidades tácitas que requiere la profesión, capacidad de renovación de conocimientos, actualización permanente y actitud crítica y objetiva.

f. La orientación de la ética profesional como apoyo para la toma de decisiones.

Se refiere a: ¿cómo cobrar lo justo, ¿cómo evitar la explotación?, razones para elegir (conocimiento moral y sentido del deber) y proceso de toma de decisiones.

g. Ética para la investigación científica y tecnológica.

Se menciona la necesidad de analizar la relación entre ciencia, tecnología y práctica y las relaciones entre la ética, la ciencia, la tecnología y la sociedad (incluyendo el medio ambiente y todos los seres vivientes).

h. Formas y metodologías sugeridas para la formación en ética profesional

Varios de los profesores indicaron que la materia debería ser optativa, que debe ser redactada por profesores o especialistas en el área ética o humanística, ofrecerse cada semestre y que ésta dependerá de la profesión o carrera que se cursa. Además se indica la necesidad de utilizar ejemplos didácticos y de la falta de ética en diferentes profesiones para mostrar su inconveniencia. Se sugiere llevar a cabo sesiones de discusión sobre lo que se considera éticamente correcto e incorrecto, análisis sobre casos reales, hacer ejercicios de auto - conocimiento en los que se puedan obtener los elementos para identificar ideales y motivaciones que llevan a los estudiantes a elegir una determinada profesión y de acuerdo con ello desenvolverse de manera positiva y productiva.

Se mencionó que el curso más que informativo debe orientarse con el objetivo de despertar la conciencia en los individuos, que a través de la lectura, el análisis y discusión de textos selectos se realcen los valores que deseamos que tengan los alumnos y los profesores y que se promuevan charlas y conferencias obligatorias para los estudiantes.

Los profesores también consideraron que ellos mismos deben conducirse con valores y destacar esto en sus clases y que en cada materia se promueva con el ejemplo la ética profesional. Algunos de ellos consideraron que "más que una materia deberían existir espacios de discusión sobre la ética" y una total transparencia sobre todos los aspectos de la investigación y la docencia. 


\section{Area 2. Ciencias Biológicas y de la Salud}

Un hecho significativo es que los posgrados de esta área aglutinan el mayor número (26.34\% del total) y el más alto grado de diversificación en las respuestas a esta pregunta abierta.

\section{De corte general y no directamente ligadas con la ética profesional}

a. Ética, Moral y Valores.

Lo encontrado en esta categoría es similar a lo ya descrito en el Área de Ciencias Físico Matemáticas y de las Ingenierías en cuanto a qué es la ética y su vinculación con otros espacios (sociedad, vida cotidiana y familia), sobre qué son los valores, su clasificación e importancia y sobre los principios morales y de la conducta humana.

No se especifican corrientes y autores de la Filosofía y se incluyen dos aspectos importantes: crisis de los valores y formación integral del ser humano.

b. Valores Cívicos y Derechos Humanos.

Incluye: justicia, justicia social, tolerancia, equidad, igualdad, dignidad de la persona humana, derechos y obligaciones humanas, derechos humanos de $1^{\mathrm{a}}$, $2^{\mathrm{a}}, 3^{\mathrm{a}}$ y $4^{\mathrm{a}}$ generación, respeto a la vida, principios básicos que norman las actividades humanas, civismo, ¿qué es y cómo funciona el amor a la patria?, responsabilidad civil, identidad nacional y la salud como un derecho humano.

Como podemos observar, además de los valores mencionados en el área 1 , los profesores de la muestra incluyeron los derechos humanos en general y la salud como uno de los principales derechos.

c. Historia, cultura y educación:

También en esta categoría, los temas expresados son similares en las dos áreas de conocimiento.

d. Conducta anticorrupción.

Se indicaron temas como los siguientes: ejemplos de grandes fraudes durante el ejercicio de la profesión, daños de la corrupción en México en el sector gobierno y privado, el plagio y sus consecuencias, lo negativo de la fabricación de datos, el papel del autoengaño en la falsificación de resultados con el fin de obtener reconocimiento social y definición de términos como fraude y falsificación.

\section{Valores vinculantes entre la ética en general y la ética profesional.}

Son principalmente: respeto, honestidad y honradez, responsabilidad, beneficencia, no maleficencia, autonomía, lealtad, libertad, constancia, confianza, liderazgo y compromiso social y ecológico.

\section{Categorías y rasgos vinculados directamente con la ética profesional}

Se reitera que en esta área se encuentra el mayor número de respuestas directamente vinculadas con la ética profesional. 
a. Ética profesional:

Su definición y papel en la sociedad actual, objetivo de ser un buen profesional, formas de desarrollo, actitudes ante la ética profesional, importancia de aplicarla, ética en el trabajo, actos profesionales y ética, profesionalismo, capacidad de producir resultados consistentes, problemas sociales en que puede incidir el profesionista, cómo se maneja un profesional en la carrera que eligió, impacto profesional y responsabilidad social, relación del profesional con su entorno, implicaciones morales y éticas de las relaciones entre colegas y en los diferentes campos de trabajo y el humanismo como eje de la acción profesional.

También se mencionaron la necesidad de definir los conceptos de profesión y práctica profesional, la historia y filosofía de la profesión y los dilemas, conflictos y errores éticos.

b. Ética profesional en las Ciencias Biológicas y de la Salud.

Se distinguen temas muy particulares de ésta área como son:

- Bioética y dignidad humana.

- Ética para el manejo de animales e implicaciones morales y éticas del impacto que tiene en la sociedad mexicana la legislación en cuanto a la producción e importación de productos de origen animal.

- Contraste entre los aspectos éticos individuales y balance con los beneficios sociales de temas morales en debate (aborto, anticoncepción del día siguiente, uso de especies transgénicas, eutanasia, etc.).

- Implicaciones del avance biotecnológico, ética para el manejo de muestras humanas, Proyecto Genoma, manipulación genética y terapia genética, clonación, producción de organismos transgénicos, transferencia horizontal de genes, estado de la regulación legal de la manipulación genética, derecho del no nacido y de los padres, uso de clones para asistencia médica de los hijos, trascendencia de la información obtenida a partir de organismos transgénicos y difusión objetiva de la información obtenida en los campos relacionados con la genética y la biotecnología.

- Uso y explotación de recursos y el impacto al ambiente.

- Con base en el marco teórico del proyecto de investigación sobre ética profesional de la UNAM, podemos decir que hay un marcado énfasis en el Principio de Autonomía de la ética profesional (HIRSCH, 2009), que tiene dos lados: la autonomía del profesional, que incluye la necesidad y posibilidad de tomar decisiones de carácter ético y la autonomía del beneficiario de la actividad profesional, que cuenta con derechos que deben ser protegidos por el profesional. En la primera acepción, los profesores de Ciencias Biológicas y de la Salud indicaron la importancia de los valores, derechos y ética profesional de los profesionistas de esta área de conocimiento (específicamente de los médicos, enfermeras y biólogos) y los campos de incidencia en donde el profesional aplica su ética profesional. 
En la segunda vertiente del Principio de Autonomía, que se refiere a los beneficiarios directos e indirectos de la actividad profesional, se expresaron las siguientes respuestas: calidad y buen trato en la atención de los pacientes, relación médico/paciente, valores y derechos de los pacientes y usuarios de los servicios de salud (específicamente también: derechos de niñas y niños, sexuales y reproductivos y de los pacientes mentales) y derecho a la información que tienen los pacientes.

c. En relación con las Competencias y habilidades sociales, se indicó lo siguiente: saber escuchar, relaciones interpersonales, manejo de conflictos interpersonales, resolución de conflictos, la comunicación como vehículo para generar consensos, colaboración, comunicación verbal y no verbal, el beneficio de trabajar juntos, trabajar en equipo, buenas relaciones estudiante-tutor, tener confianza en los demás, disciplina y puntualidad.

d. En la categoría de Legalidad, normatividad y principios y reglas de la ética profesional, están: deontología, normas de conducta, códigos de ética, aspectos legales que norman el ejercicio de la misma, Ley General de Salud y necesidad de respetar los reglamentos y someterse a sus normas.

e. En los tres rubros de respuesta sobre identidad, encontramos lo siguiente:

En identidad en general se incluye autocrítica, autoestima y motivación a la excelencia personal. En identidad institucional, están: ética y universidad, el papel de la universidad en el proceso de formación de ciudadanos críticos y contribuir al debate de ideas acerca de la universalidad y colegialidad de la universidad. En identidad profesional se integraron: vocación, el no buscar reconocimiento sino trabajar por el amor a la profesión y temas que induzcan a entender "lo importante de seleccionar un trabajo que te satisfaga y aprender a tomar gusto por lo que haces".

f. En Rasgos de las competencias cognitivas se ubicaron: riesgos del monopolio del saber, crisis de la racionalidad técnica, superación profesional, actualización, criterio, rigor académico, reconocer errores y cómo aprender de ellos, hacer bien las cosas, creatividad, epistemología, visión de futuro, la diferencia entre sólo pasar una materia y obtener conocimiento para resolver problemas, nuevas perspectivas en el siglo XXI e interrelación con otras profesiones y especialidades.

g. El rubro de Orientación de la ética profesional como apoyo para la toma de decisiones se refiere especialmente a las implicaciones sociales de la toma de decisiones.

f. En Ética para la investigación científica y tecnológica colocamos las siguientes respuestas: respetar la ética científica en las publicaciones, ciencia y sociedad, importancia de la verdad en el desarrollo de la ciencia, ética de la ciencia y la tecnología, desarrollo de la ciencia y sus riesgos, la necesidad de estar informado y de tomar una posición con respecto a los avances en la ciencia y la tecnología e implicaciones éticas, legales y sociales de los avances científicos y tecnológicos de cada campo. 
h. Formas y metodologías sugeridas para la formación en ética profesional

Se indicó la posibilidad de incluir estudios de caso sobre comportamientos éticos de individuos e instituciones y de comportamientos no éticos, casos reales e hipotéticos y opciones sobre la actuación, una logística que involucre a los alumnos en el ejercicio de su profesión con las problemáticas de su comunidad y un taller con ejercicios que provoquen el conflicto de decisiones.

Entre las opciones de formación que no incluyen una materia de ética profesional están:

"Si el profesor tiene una buena formación ética, esta se transmitirá a los estudiantes, sin la necesidad de que haya un curso específico" y "las actitudes éticas no se aprenden en aula, sino que resultan del entorno".

\section{Área 3. Ciencias Sociales}

\section{De corte general y no directamente ligadas con la ética profesional}

a. Ética, Moral y Valores.

Las respuestas incluyen muchos de los aspectos de las otras dos áreas sobre la ética en general, normas morales y valores éticos y algunas cuestiones específicas como: lectura de grandes pensadores como Platón y Aristóteles y de los clásicos y contemporáneos de la ética y ética ontológica y deontológica. También se mencionaron las consecuencias de la falta de ética, ética en la interdisciplina, multidisciplina y transdisciplina, ética como columna vertebral del desarrollo social y análisis de problemas morales específicos (aborto, drogadicción, corrupción, eutanasia, etc.). Se encuentran también respuestas ligadas con el compromiso social y ecológico.

b. En la categoría de Valores Cívicos y Derechos Humanos se incluyen (al igual que en las otras dos áreas previamente descritas) los derechos humanos y diversos valores específicos (por ejemplo: libertad, solidaridad, tolerancia, lealtad, justicia, humildad y equidad).

c. En Historia, Cultura y Educación, del mismo modo que en las dos áreas anteriores, también están aspectos muy diversos a nivel mundial y de México.

d. En Conducta anticorrupción se mencionaron: aspectos legales del fraude profesional y de la usurpación de funciones y corrupción y Estado en México.

2. Los Valores vinculantes entre la ética en general y la ética profesional fueron: responsabilidad y compromiso, honestidad, respeto, integridad y autonomía.

\section{Categorías y rasgos vinculados directamente con la ética profesional}

a. Ética profesional.

Incluye el mismo tipo de respuestas generales de las otras dos áreas sobre la ética profesional y también se enfatiza el compromiso social, las implicaciones del ejercicio profesional y con respecto a diferentes grupos (vulnerables, margina- 
les, menores de edad, etc.). Llama la atención que haya referencia a temas de las Ciencias Biológicas y de la salud, con respecto a la Bioética, la investigación para resolver problemas prioritarios de salud y de ética clínica. Este tipo de respuestas muy probablemente provienen de los profesores del Posgrado en Psicología.

b. Las Competencias y habilidades sociales mencionadas son: disciplina en el trabajo, puntualidad, importancia del trabajo en equipo, valorar el trabajo y el tiempo de otros así como el propio, colaboración, importancia de la comunicación como clave en la transmisión de valores, resolución de conflictos y relaciones humanas y convivencia social.

c. En la categoría de Legalidad, normatividad y principios y reglas de la ética profesional, se mencionaron: códigos de las profesiones y de ética en la práctica de la enseñanza y en investigación y difusión de las ciencias, aspectos legales relacionados al ejercicio de la profesión, conciencia de que toda norma jurídica contiene un sustrato ético, derechos de autor y los derechos de los alumnos y profesores.

d. Identidad personal incluye lealtad y fidelidad hacia uno mismo, consideraciones en torno al cuidado de uno mismo, la personalidad y la esencia como integrantes del ser humano, desarrollo de la autoconciencia, identidad y pertenencia al grupo y perspectiva individual. En identidad institucional se sintetizaron: difusión de los derechos universitarios, instituciones sociales y valores, identificación y valoración de la educación pública, en especial de la UNAM y valorar el costo de oportunidad social de los recursos invertidos en la educación. En identidad profesional, están: privilegio de ser un profesionista, vocación, identificación del fracaso y logro profesional y valoración del trabajo.

e. En cuanto a los Rasgos de las competencias cognitivas se integraron respuestas sobre: cómo valorar el precio y el costo de los conocimientos para la formación profesional, inteligencia, orientaciones para la formación del juicio crítico y de la autocrítica, inculcar la búsqueda de la superación personal en todos los niveles, elementos para la conformación de equipos interdisciplinarios y calidad en la formación.

f. En el rubro de Orientación de la ética profesional como apoyo para la toma de decisiones el tema más mencionado fue el de los dilemas éticos.

g. En Ética para la investigación científica y tecnológica se expresaron las siguientes ideas: consecuencias de los resultados de investigación y divulgación del conocimiento científico de manera responsable.

h. Formas y metodologías sugeridas para la formación en ética profesional

- Debe estar acorde al plan de estudios de cada carrera.

- El contenido no debe ser moralizante, sino un estudio serio de la ética como parte de la Filosofía y de los profesionales de la carrera en cuestión.

- Lo que determine una comisión multidisciplinaria. 
- La orientación temática debe tener no sólo un sentido filosófico abstracto que la hace a veces de difícil comprensión, sino una visión práctica aplicable a casos concretos con el fin de motivar claramente al alumno a aplicar los principios expuestos y a evitar sus omisiones.

- Un desarrollo breve y sistemático, por unidades, incisos y subincisos de manera que sea totalmente comprensible y aplicable con planteamientos de casos concretos y con la participación activa del alumno. Ejercicios prácticos de discusión, reflexión y análisis y de propuesta de soluciones creativas a los problemas actuales.

- Elaboración de un código de ética profesional aplicado a la profesión. Inclusión de dilemas éticos.

- Dinámicas en grupo y fuera del aula. Considerar el contexto en dónde se requiere tomar decisiones éticas.

- Lecturas obligatorias (se indicaron numerosos autores).

- Formación de profesores.

- Distinguir entre la ética y la ética profesional.

También hubo muchas respuestas de no incluir una materia de ética profesional, por ser el profesor un modelo de conducta, por tratarse de asuntos de conducta personal y no de contenidos académicos, debido a que la ética se aprende en las actividades cotidianas, porque requiere ser trabajo colegiado y porque deberían ser contenidos transversales.

\section{Area 4. Humanidades y de las Artes}

\section{1) De corte general y no directamente ligadas con la ética profesional}

a. Ética, Moral y Valores

En las tres áreas descritas y también en ésta se presentan las mismas ideas sobre la ética en general, la moral y la ética y los valores. En las otras áreas, en esta categoría, se hace referencia explícita a la Filosofía y a la Axiología. Lo mismo acontece en la cuarta, pues se indica la necesidad de enseñar ética filosófica, sus posturas y paradigmas, autores, modelos y métodos.

b. En la categoría acerca de los Valores Cívicos y Derechos Humanos, se incluyen también los derechos humanos, el estudio de los derechos, las obligaciones y las responsabilidades del ser humano; libertad, justicia, solidaridad, tolerancia, trato digno, respeto a la diversidad, civismo, reconocer los valores patrios, liderazgo en el país, cultura ciudadana, amor al país, integridad individual y social, centralidad de la vida y dignidad humanas y una visión integral de la actividad humana. se repiten.

c. En Historia, Cultura y Educación los temas de las otras áreas también

d. En Conducta anticorrupción se indicó el rechazo a la corrupción. 


\section{Valores vinculantes entre la ética en general y la ética profesional.}

Se explicitaron: respeto, honestidad, responsabilidad, servicio, compromiso y sentido del deber. También hubo numerosas menciones al compromiso social y ecológico.

\section{Categorías y rasgos vinculados directamente con la ética profesional}

\section{a. Ética profesional.}

Fueron muy numerosas las respuestas que se incluyen en esta categoría y son similares a las de las demás áreas.

Específicamente sobre el campo de conocimiento de esta área se menciona: que el alumno comprenda las obligaciones y deberes del arquitecto con la sociedad que va a servir y a la que le va a construir su hábitat y el conocimiento de los reglamentos de construcción vigentes. En el caso de los historiadores del arte y los historiadores se enfatizó que debe haber un fuerte compromiso ético en el manejo de las fuentes documentales y los objetos de estudio, un compromiso con el otorgamiento de créditos a las ideas tomadas de otros, un respeto por la diversidad en la opinión sobre los mismos temas y un compromiso con el apoyo a los alumnos y a la formación de investigadores jóvenes.

b. En Competencias y habilidades sociales, se especifican: desarrollo de habilidades en la comunicación interpersonal y de trabajo en grupo, cooperación entre colegas, cumplimiento de tareas y horarios, relaciones humanas, reciprocidad, relaciones profesionales y habilidades para la comprensión del entorno y de la realidad objetiva que lo rodea.

c. Acerca de Legalidad, normatividad y principios y reglas de la ética profesional se indicaron: conocimiento de la estructura de contratos de trabajo profesional, códigos de ética profesional, leyes que existen en caso de incumplimientos en la profesión, estudio de las normas profesionales, análisis de la pertinencia de la normatividad vigente, derechos y obligaciones con base en lo establecido en la Constitución Política, la legislación de la UNAM y reglamentos que aplican para ejercer la práctica profesional, respeto a las leyes, veracidad y las distintas formas de reflexión ética acerca de las normas morales, religiosas o jurídicas que rigen a la sociedad.

d. En Identidad personal se agrupa: autoconocimiento, auto - confianza, valoración de uno mismo, asertividad, individualidad, reconocimiento de su importancia como persona, reconocimiento de los alcances y límites personales y felicidad. En identidad institucional están: ética universitaria, compromiso con los valores universitarios y la vida institucional, fomentar la identidad universitaria como institución educativa, hermandad universitaria, la universidad, la UNAM y los universitarios, señalar la relación de la UNAM con la sociedad mexicana, logística universitaria y conocer algunos pensadores que han egresado de la UNAM.

Las respuestas sobre identidad profesional fueron: identidad y conocimiento de la profesión, principios de vocación de servicio y lealtad a la profesión. 
e. En los Rasgos de las competencias cognitivas se manifiesta: transdisciplina, pensamiento complejo, autocrítica, creatividad, reflexión, estar informados, ser pensadores, preparación y actualización, innovación, superación académica, conciencia de tener más y mejores conocimientos, desarrollo de habilidades en la lectura y conducta lectora para su auto superación, conocimiento y pragmatismo, principios y prácticas de reflexión, entendimiento y diálogo respecto de la construcción de las propias ideas y las de los demás, importancia (instrumental y en sí misma) del conocimiento objetivo y riguroso, racionalidad, teoría y práctica, conocimiento del área de estudio en el campo profesional, superación personal y retos.

f. Sobre la Orientación de la ética profesional como apoyo para la toma de decisiones, al igual que en las otras áreas se hace referencia a toma de decisiones constructivas, análisis de qué acciones por parte del profesional ponen en riesgo a la sociedad y dilemas éticos.

g. Son muy pocas las referencias directas acerca de la Ética para la investigación científica y tecnológica y se refieren al cuestionamiento sobre qué es la ciencia y a la necesidad de contar con contenidos de introducción a la ética para la investigación científica.

Los enfoques disciplinarios específicos son: la semiología, la acción moral de los sujetos y las valoraciones de las conductas de acuerdo con el liberalismo y el determinismo, movimientos sociales en la historia, Historiografía crítica, bases históricas y contemporáneas de la Psicología y la Sociología y sus interrelaciones, análisis comparativo del desarrollo de los pueblos visto histórica y contemporáneamente, desarrollo histórico de los sistemas políticos y la crítica que se puede hacer en la actualidad a su desempeño moral, social, intelectual y productivo y el papel de la filosofía en el desarrollo de la humanidad. sional son:

h. Las Formas y metodologías sugeridas para la formación en ética profe-

- Talleres de estudios de casos, relacionar con la vida diaria y ejemplos prácticos, argumentar racionalmente y poder sostener una controversia de modo racional sobre diferentes posiciones éticas y toma de decisionessoluciones.

- Seminario de problemas contemporáneos dónde se discutiera cómo resolver estos asuntos desde varios puntos de vista.

- "Una pequeña proporción de conceptos teóricos y filosóficos y una gran proporción de análisis crítico de la práctica profesional”.

- "Más que los contenidos de la materia, es clave elegir un buen maestro que imparta bien y transmita los valores y se constituya en modelo".

- "Sería sólo para transmitir las normas éticas de la profesión, ya que los valores no se enseñan a través de estrategias educativas, sino por medio del ejemplo cotidiano en las acciones de los individuos". "Es un eje que debería atravesar la universidad y la sociedad". 
- Crear actividades de participación comunitaria en la que se solicite una participación de los alumnos, las cuales deben estar encaminadas a conocer la problemática social, sensibilizarse ante la misma y ver la forma en que el desarrollo profesional sea de utilidad a la sociedad.

\section{Conclusiones}

- La pregunta abierta acerca de si los profesores de posgrado consideran que debería haber una materia sobre ética profesional para todos los estudiantes resultó muy exitosa; cuestión que puede notarse fácilmente por la cantidad y diversidad de información que se describe y analiza en el artículo.

- Es evidente la dificultad de muchos de los profesores de posgrado en centrarse en respuestas directamente relacionadas con la ética profesional, pues muchas de ellas se ubican claramente en la ética general. Podemos suponer, que esto puede deberse a que tengan poco conocimiento sobre el campo temático de la ética profesional.

- En las respuestas ligadas con la identidad institucional, es fácil notar una fuerte vinculación de los profesores con la UNAM.

- En esta pregunta, las competencias sociales no salieron tan bajas como en el caso de la pregunta abierta sobre cuáles son los cinco principales rasgos de "ser un buen profesional" y en la escala de actitudes sobre ética profesional, que se aplicaron a la misma muestra de profesores de posgrado de la UNAM.

- Por el contrario, las competencias cognitivas obtuvieron bajos porcentajes en esta pregunta, a diferencia de la pregunta abierta sobre los cinco rasgos de "ser un buen profesional" y la escala.

- El compromiso social y ecológico, que salió con bajos puntajes en la pregunta abierta sobre cuáles son los cinco principales rasgos de "ser un buen profesional" y en la escala de actitudes, adquirió mayor importancia en la pregunta que se desarrolla en el artículo acerca de la necesidad y los contenidos de una materia de ética profesional.

- Resultaron muy interesantes las respuestas referidas al mal comportamiento ético.

- La mayor parte de los profesores coincide en la necesidad de formar en ética profesional a los estudiantes, con base en metodologías activas y mostrando las consecuencias que tienen y que pueden tener los errores éticos que se cometen.

- Con respecto a las respuestas sobre ética profesional por área de conocimiento, sobresale la de Ciencias Biológicas y de la Salud, que además de haber proporcionado el mayor número de contestaciones a esta pregunta, indica temas específicos que se desarrollan en los posgrados. En Ciencias Sociales también se mencionan temas vinculados con la salud y hay re- 
ferencias acerca de la protección especial a los grupos marginados y en vulnerabilidad. Resultó interesante la indicación - en esta última área- de algunos profesores en la necesidad de distinguir con claridad la ética en general y la ética profesional. Los profesores de Ciencias Físico Matemáticas y de las Ingenierías proporcionaron el menor número de respuestas.

\section{Notas}

${ }^{1}$ Además de los conocimientos y habilidades básicos para el ejercicio de cada profesión, pueden incluirse áreas de desarrollo sobre la ética profesional en tres niveles: ética general de las profesiones, ética profesional de las grandes áreas del conocimiento y ética profesional específica de cada una de las disciplinas universitarias. Otros dos aspectos que se relacionan estrechamente, son la ética de la docencia y la ética de la investigación científica.

2 Para Augusto Hortal (2002, p. 29) entre la ética general que se ocupa de la reflexión acerca de los elementos constitutivos de la vida moral y las actuaciones puntuales, están las éticas aplicadas. Ahí se ubican la ética general de las profesiones y la ética de cada profesión en particular. Las éticas aplicadas; entre ellas, las éticas profesionales, tratan de especificar los criterios que pueden orientar un ámbito particular de las actividades humanas. Su objetivo es proporcionar los elementos que se requieren para conformar una conducta ética habitual en el complejo y diversificado espacio del ejercicio profesional.

${ }^{3}$ El marco teórico consta de los siguientes aspectos: importancia de la ética profesional para las instituciones de educación superior, definiciones y caracterización de profesión en general y de ética profesional en particular, principios, competencias y reglas de la ética profesional, ética profesional como parte de las éticas aplicadas, diferencia entre ética profesional y deontología profesional, influencias económicas, técnicas y organizacionales en el ejercicio profesional, valores fundamentales, especialmente responsabilidad; identidad profesional, ética de la docencia, ética de las ciencias y de la investigación científica y propuestas de formación valoral en este campo.

${ }^{4}$ En el 2006 también se generó el Proyecto Interuniversitario sobre Ética Profesional, en el que participan otras 15 universidades mexicanas. Los equipos que se formaron en cada una de estas instituciones utilizaron, en su mayoría, la misma estrategia metodológica para encuestar simultáneamente a estudiantes y profesores de posgrado.

${ }^{5}$ De los 719 profesores de la muestra, sólo 704 contestaron la pregunta abierta sobre sí debería haber una materia de ética profesional para todos los estudiantes.

${ }^{6}$ Un académico no especificó el o los posgrados donde imparte clases y tutorías.

${ }^{7}$ En Ciencias Físico Matemáticas y de las Ingenierías están los siguientes siete posgrados: Astronomía, Ingeniería, Ciencia e Ingeniería de la Computación, Ciencia e Ingeniería de Materiales, Ciencias de la Tierra, Ciencias Físicas y Matemáticas.

${ }^{8}$ En Ciencias Biológicas y de la Salud se ubican los siguientes nueve posgrados: Ciencias Biológicas, Ciencias Biomédicas, Ciencias Bioquímicas, Ciencias del Mar y Limnología, Ciencias Médicas - Odontológicas y de la Salud; Neurobiología, Ciencias de la Producción y de la Salud Animal, Ciencias Químicas y Enfermería.

${ }^{9}$ En Ciencias Sociales hay nueve posgrados: Antropología, Ciencias de la Administración, Ciencias Políticas y Sociales, Derecho, Economía, Estudios Latinoamericanos, Geografía, Psicología y Trabajo Social.

${ }^{10} \mathrm{En}$ el Área de Humanidades y de las Artes están trece posgrados: Arquitectura, Artes visuales, Bibliotecología y Estudios de la Información, Estudios Mesoamericanos, Filosofía, Filosofía de la Ciencia, Historia, Historia del Arte, Letras, Lingüística, Música, Pedagogía y Urbanismo. 


\section{REFERENCIAS}

ALCÁNTARA, A.; BARBA, L.; HIRSCH, A. Valores universitarios y profesionales de los estudiantes de posgrado de la UNAM. México: Instituto de Investigaciones sobre la Universidad y la Educación - UNAM, 2009.

HIRSCH, A. Principios y reglas de la ética profesional para el Proyecto Interuniversitario sobre Ética Profesional en México. En: IBÁÑEZ - MARTÍN, J. A. (Coord.). Educación, Conocimiento y Justicia. Madrid - España: Dykinson, S.L., 2009. p. 321-331.

HORTAL, A. Ética General de las Profesiones. Bilbao, España: Desclée De Brouwer, 2002.

UNAM. Dirección General de Estudios de Posgrado. Programas de Posgrado.

Recebido em: novembro de 2008

Aceito em: outubro de 2009 